\title{
From the Editors' Desk: Medicine as a Service Industry
}

\author{
Richard L. Kravitz, MD, MSPH \\ Division of General Medicine, UC Davis, Sacramento, CA, USA.
}

$\mathrm{J}$ Gen Intern Med 25(4):279

DOI: $10.1007 / \mathrm{s} 11606-010-1282-4$

(c) Society of General Internal Medicine 2010

Health care is a service industry. However, despite decades of effort, the delivery of health care is organized to serve the needs of providers and staff rather than patients. Other service industries do a far better job. Imagine the scene if Starbucks performed like many health care clinics.

Customer: (Trying to catch eye of Barista, who is staring at computer screen.) Uh hem.

Barista: Good morning. How can I help you?

Customer: I'd like a latte.

Barista: (Sullenly.) Do you have an appointment?

Customer: No. I was hoping someone could fit me in.

Barista: Starbucks Record Number?

Customer: (Fumbling through wallet.) Let's see. Oh here it is. 157-08-29.

Barista: Ok, Frederick. Still on Adams Park Lane?

Customer: Yes.

Barista: CoffeeWorks insurance?

Customer: Yes.

Barista: Did you know you owe us a copay?

Customer: Really? I'm sure I took care of that last time.

Barista: Well, you can settle later. (Typing) I can get you in for a latte at 10:45. Please have a seat.

Customer: 10:45? That's almost 2 hours from now. Don't you have something sooner?

Barista: Next time make an appointment.

Customer: I tried to make an appointment, but I kept getting routed into voicemail.

Barista: Well, Frederick, this is coffee we are talking about. Shouldn't that be a priority?

In this issue of JGIM, Ly and Glied report on data from several waves of the Commmunity Practice Study (CPS) Household Survey. The CPS is an ongoing longitudinal study of access, quality, and costs of health care in 60 US communities, coordinated by the Center for Studying Health System Change. In their study of over 40,000 sick visits to primary care physicians, patients waited an average of about

Published online March 4, 2010
5 days for an appointment; once in the waiting room, they waited nearly $30 \mathrm{~min}$ to see the doctor. Waiting times of this magnitude are not only difficult to square with any reasonable standard of patient-centeredness, they are arguably inefficient, as 5 days is enough time for some acute problems to evolve from minor to severe. From a different perspective, one might ask whether all these office visits are necessary in the first place. Our visit-centered approach to handling acute and chronic problems follows directly from the current visitbased reimbursement system and represents a huge misdirection of resources. In any case, the systems we have in place to dispense visits could arguably take a lesson from Starbucks (the real one).

In addition to documenting a lamentable level of service overall, the article by Ly and Glied highlights several troublesome racial and ethnic disparities. Blacks and Hispanics waited longer for an appointment than white patients, and once in the waiting room, they sat there longer. The differences were not explained by geography, although the tendency of minorities to use different providers than whites could not be excluded. It is well know that minority patients use a different (albeit overlapping) mix of providers, and these usage patterns can have both beneficial and deleterious effects. For example, the RAND evaluation of Medicare's Prospective Payment System found that black patients received equivalent quality of care compared with whites overall, but only because African-American elders tended to be admitted to urban teaching hospitals, where quality of care was higher. When analyzed at the hospital level, blacks received the same or worse care as whites.

Even as prospects for comprehensive health reform appear uncertain, momentum is mounting for comprehensive health care delivery reform that includes improvements in service. Making this happen will require changes in policy and payment, to be sure. But the greatest gains may come from further evolution in the culture of medicine towards patientcentered, customized, service-oriented care.

Half-caff, double-shot, skim-milk venti latte with a shake of cinnamon, anyone?

Corresponding Author: Richard L. Kravitz, MD, MSPH, Division of General Medicine, UC Davis, 4150 V. Street, Suite 2400 PSSB, Sacramento, CA 95817, USA (e-mail: rlkravitz@ucdavis.edu). 